\title{
Mesoscale Eddy Energy Locality in an Idealized Ocean Model
}

\author{
IAN Grooms, Louis-Philippe NADEAU, AND K. SHAFER SMITH \\ Center for Atmosphere Ocean Science, Courant Institute of Mathematical Sciences, New York, New York
}

(Manuscript received 13 February 2013, in final form 6 May 2013)

\begin{abstract}
This paper investigates the energy budget of mesoscale eddies in wind-driven two-layer quasigeostrophic simulations. Intuitively, eddy energy can be generated, dissipated, and fluxed from place to place; regions where the budget balances generation and dissipation are "local" and regions that export or import large amounts of eddy energy are "nonlocal." Many mesoscale parameterizations assume that statistics of the unresolved eddies behave as local functions of the resolved large scales, and studies that relate doubly periodic simulations to ocean patches must assume that the ocean patches have local energetics. This study derives and diagnoses the eddy energy budget in simulations of wind-driven gyres. To more closely approximate the ideas of subgrid-scale parameterization, the authors define the mean and eddies using a spatial filter rather than the more common time average. The eddy energy budget is strongly nonlocal over nearly half the domain in the simulations. In particular, in the intergyre region the eddies lose energy through interactions with the mean, and this energy loss can only be compensated by nonlocal flux of energy from elsewhere in the domain. This study also runs doubly periodic simulations corresponding to ocean patches from basin simulations. The eddy energy level of ocean patches in the basin simulations matches the level in the periodic simulations only in regions with local eddy energy budgets.
\end{abstract}

\section{Introduction}

Mesoscale eddies are a ubiquitous feature of ocean dynamics and have been the subject of myriad investigations. Gill et al. (1974) showed that the potential energy of the large-scale-mean circulation is much greater than its kinetic energy and argued that the conversion of large-scale available potential energy by baroclinic instability is primarily responsible for the ubiquity of mesoscale eddies. Diagnostic studies of eddy energetics in numerical simulations began in the 1970s; Harrison (1979) reviews the results. Early attention (e.g., Holland 1978) was focused on the partition of energy between kinetic and potential, mean and eddy, and on domain-integrated budgets. A notable exception is Harrison and Robinson (1978), who analyzed the energy budget over a few subregions of a single-gyre basin. Subsequent work by Hall (1986) and Treguier (1992) also analyzed the energy budget over subregions of their computation domains. Each of these energy budget analyses demonstrates that the eddy energy

Corresponding author address: Ian Grooms, Center for Atmosphere Ocean Science, Courant Institute of Mathematical Sciences, New York University, 251 Mercer St., New York, NY 10012. E-mail: grooms@cims.nyu.edu budget of an ocean patch balances local generation, local dissipation, and nonlocal import or export of energy. However, none of these analyses directly addresses the question of energy locality, namely, the extent to which local eddy generation is balanced by local eddy dissipation.

The question of eddy energy locality bears on the subject of mesoscale parameterization because the effects of unresolved mesoscale eddies on a resolved mean flow depend on the eddy energy. Visbeck et al. (1997) and Cessi (2008) both relate the transfer coefficient $\kappa$ in the Gent and McWilliams (1990) parameterization to eddy energy using a mixing-length approximation $\kappa \approx v_{e} l_{e}$ where $l_{e}$ is a mixing length and $v_{e}$ is an eddy velocity dependent on the eddy energy. These parameterizations are local in the sense that $v_{e}$ and $l_{e}$ are functions only of the local values of the resolved, large-scale variables and of external parameters like the strength of bottom friction and the local Coriolis frequency. ${ }^{1}$ In contrast, Eden and Greatbatch (2008) and Marshall and Adcroft (2010)

\footnotetext{
${ }^{1}$ The parameterizations of Visbeck et al. (1997), Cessi (2008), and Ferrari et al. (2010) are nonlocal vertically; "horizontally" is implicit in our use of local.
} 
propose parameterizations that allow for nonlocality in the eddy energy budget by solving prognostic equations for eddy energy on the coarse grid. A recent multiscale asymptotic analysis by Grooms et al. (2012) shows that nonlocal components, including an advective flux of energy from one place to another, can be comparable to local generation and dissipation in the eddy energy budget.

Energy locality is also important in relating the results of doubly periodic simulations to patches of ocean. Because doubly periodic simulations always have local energetics, they are unable to correctly model patches of ocean that have nonlocal energetics. For example, Arbic and Flierl (2004b) and Arbic and Scott (2008) make inferences about the type and strength of bottom friction operative in the oceans by comparing doubly periodic simulations to observational data. The relevance of these results is contingent on the energetic locality of the observed eddies.

The question of locality is also connected to the results of doubly periodic simulations with imposed nonzonal baroclinic shear on a $\beta$ plane. Doubly periodic simulations with imposed nonzonal baroclinic shear often develop unrealistically large energy levels (Arbic and Flierl 2004a; Smith 2007). A simple explanation of the mechanism is that the flow, under the influence of $\beta$, tends to organize into zonal jets that extract energy from imposed nonzonal shear more efficiently than from imposed zonal shear. One possible explanation for the unrealistically high energy levels in doubly periodic simulations with nonzonal shear is that the eddy energy budget in these ocean patches is nonlocal: rather than being balanced by dissipation, efficient eddy energy generation in these patches could be balanced by a net export of energy, which is lacking in doubly periodic simulations.

Venaille et al. (2011) investigate locality by comparing eddies from ocean patches in a general circulation model (GCM) with eddies in doubly periodic quasigeostrophic (QG) simulations. Though able to demonstrate locality by agreement between the QG and GCM eddies, this approach has difficulty in conclusively demonstrating nonlocality because any failure of the QG simulations to match their GCM counterparts can potentially be attributed to a mismatch in the dynamics ( $Q G$ versus primitive equations) rather than to nonlocality per se.

We address the question of energy locality of mesoscale eddies by diagnosing the energy budget of eddies in simulations of two-layer quasigeostrophic dynamics in a wind-driven basin configuration. We say that the eddy energy budget is "local" if it comprises a balance between local energy generation (through interaction with the mean flow, wind forcing, etc.) and dissipation.
This question is formally distinct from the parameterization question of whether the eddy fluxes of momentum and tracers are local functions of the large scale, which we do not address directly; we simply assume that nonlocality in the eddy energy budget suggests some degree of nonlocality in the fluxes.

There are many ways of defining "mean" and "eddy." In the context of energetics, the most popular definition of the mean is the time average. This has several advantages, including analytical tractability and ease of implementation. But in the context of mesoscale ocean dynamics, and in particular in the context of parameterization, the concepts of mean and eddy are more intuitively connected to spatial scale than to a time average, and the time-average definition of mean and eddies is only indirectly connected to spatial scale. For example, time-mean analysis allows nonstationary largescale features, like fast barotropic Rossby waves or oscillating gyres, to appear in the eddy field and small-scale standing features, like thin stationary jets, to appear in the mean. In addition, topographic interactions and Rossby wave reflection at boundaries are able to transfer energy from large to small scales, but in the time-mean analysis topography and boundaries cannot mediate energy transfer between the mean and eddies.

In zonal channels and doubly periodic domains, the zonal mean is often preferred to a time average for defining mean and eddies. As with the time average, this has advantages in terms of computational and analytical tractability, but the zonal-mean analysis allows features with small zonal scales (e.g., jets) to appear in the mean and features with large meridional scales (e.g., largescale vortices) to appear as eddies. These disadvantages distance the results of time-mean and zonal-mean analyses from the spatial-scale concept of mean and eddies and particularly from the application of subgrid-scale parameterization.

In addition to deviations from a zonal mean or time mean, the term eddy can also refer to nonlinear coherent vortices; indeed, it is natural to think of these as the eddies regardless of the definition of the mean. Analysis of large altimetric datasets shows that these eddies typically propagate westward over long distances (Chelton et al. 2007, 2011), which suggests that the eddy energy budget is nonlocal. But eddy propagation only suggests energetic nonlocality and does not necessarily imply it. If, for example, an eddy enters and leaves a quiescent region without significantly changing amplitude, then its passage has no effect on the time-averaged energy budget for that region. Similarly, in the case of eddy formation or lysis, the energy flux associated with a coherent eddy entering or leaving a region can be balanced by a flux of incoherent small-scale eddy energy. Thus, the formation, 
propagation, and decay of nonlinear coherent vortical eddies can only be viewed as suggestive of energetic nonlocality-an intuition that requires confirmation.

To avoid the difficulties inherent in the time-mean and zonal-mean formulations, we use a purely spatial filter of the type used by Nadiga (2008); we are unaware of other local energetic analyses of mesoscale eddies that define the mean by a spatial filter. ${ }^{2}$ We also address the question of locality by comparing doubly periodic simulations to patches of ocean in basin simulations. Venaille et al. (2011) forced doubly periodic quasigeostrophic simulations with mean baroclinic shear profiles taken from patches of ocean in a primitive equation simulation and compared the results with the eddies in the primitive equation patches. Many of the doubly periodic simulations developed excess eddy energy (suggesting nonlocal behavior) but the need to tune the balanced quasigeostrophic simulations to approximate the primitive equation dynamics meant that disagreement between the QG and primitive equation simulations could not be unambiguously attributed to nonlocal effects. Our doubly periodic simulations use the same governing quasigeostrophic dynamics as the basin and channel simulations, thereby avoiding this difficulty. As expected, we find that the energy level of the quasigeostrophic simulations correlates with the eddy energy in the ocean patches when the patches have local energetics.

As noted above, there is reason to expect that the eddy energy budget is nonlocal in regions of nonzonalmean shear, because doubly periodic simulations with nonzonal shear are often unrealistic. Some regions of nonzonal shear in our simulations do have nonlocal eddy energy budgets, in particular those at the eastern edges of the wind-driven gyres, where doubly periodic simulations bear essentially no resemblance to these ocean patches. However, other regions of nonzonal shear have local eddy energy budgets and are modeled well by doubly periodic simulations.

The outline of the paper is as follows. We present the eddy energy budget of two-layer quasigeostrophic equations based on a spatial filter in section 2 , with the details of the derivation sequestered in an appendix. In section 3 we describe the experimental setup, including the details of the code, basin dimensions, etc. In section 4, we present and analyze the results of the energetic analysis and of the comparison with doubly periodic simulations. We conclude in section 5 .

\footnotetext{
${ }^{2}$ Nadiga and Straub (2010) use a spatial filter defined by Fourier truncation to diagnose domain-integrated energetics, but such a filter is unable to produce a meaningful local energetic analysis.
}

\section{Eddy energy equations (analysis/theory)}

We analyze the energetics of the following two-layer QG equations:

$$
\begin{aligned}
\partial_{t} q_{1}+\nabla \cdot\left(\mathbf{u}_{1} q_{1}\right)+\beta v_{1}=F_{w}+A_{h} \nabla^{4} \psi_{1}, \\
\partial_{t} q_{2}+\nabla \cdot\left(\mathbf{u}_{2} q_{2}\right)+\beta v_{2}=-r \nabla^{2} \psi_{2}+A_{h} \nabla^{4} \psi_{2}, \\
q_{1}=\nabla^{2} \psi_{1}+F_{1}\left(\psi_{2}-\psi_{1}\right)-F_{0} \psi_{1}, \quad \text { and } \\
q_{2}=\nabla^{2} \psi_{2}+F_{2}\left(\psi_{1}-\psi_{2}\right),
\end{aligned}
$$

where $F_{0}=f_{0}^{2} /\left(g H_{1}\right), F_{1}=f_{0}^{2} /\left(g^{\prime} H_{1}\right)$, and $F_{2}=f_{0}^{2} /\left(g^{\prime} H_{2}\right)$. The velocity is $\mathbf{u}_{i}=\left(-\partial_{y} \psi_{i}, \partial_{x} \psi_{i}\right)$, and $F_{w}$ denotes wind forcing. The potential vorticity in each layer is $q_{i}(i=1$ is upper and $i=2$ is lower), the streamfunction is $\psi_{i}$, the depth of each layer is $H_{i}$, the local Coriolis frequency is $f_{0}$, the gravitational acceleration is $g$, and reduced gravity is $g^{\prime} ; A_{h}$ is the turbulent viscosity coefficient, $r$ is the Ekman drag coefficient, and $\beta$ is the meridional gradient of planetary vorticity. We include the effect of a free surface through the inclusion of nonzero $F_{0}$ (which is much smaller than $F_{1}$ and $F_{2}$ ), but this has minimal impact on the dynamics because the external deformation radius is close to the domain scale. The internal (baroclinic) and external (barotropic) deformation radii are the reciprocal of the square root of the eigenvalues of the matrix $\left[\left(F_{1}+F_{2}\right)-F_{1} ;-F_{2} F_{2}\right]$ (in MATLAB notation); the larger radius is barotropic. We use Laplacian vorticity diffusion rather than the more common biharmonic vorticity diffusion for reasons discussed below. The values taken by the parameters in our simulations are discussed in section 3 .

We define the large-scale-mean potential vorticity to be the solution of the following elliptic problem:

$$
\left(1-L_{f}^{2} \nabla^{2}\right) \bar{q}_{i}=q_{i}
$$

with boundary condition $\bar{q}_{i}=0$. The filter scale is $L_{f}$ : larger scales are mean and smaller scales are eddies. This is essentially the same as the filter used by Nadiga (2008). The eddy potential vorticity is defined by $q_{i}^{\prime}=q_{i}-\bar{q}_{i}$. The overbar and prime notation are defined as above for any dependent variable, not just potential vorticity.

Some insight into the behavior of the filter may be gained by examining its behavior in a periodic domain. In a periodic domain, application of the low-pass filter is equivalent to scaling the Fourier coefficients of $q_{i}$ by $\left(1+L_{f}^{2} k^{2}\right)^{-1}$, where $k$ is the modulus of the wavenumber. Large wavenumbers with $L_{f} k \gg 1$ are damped approximately as $\left(L_{f} k\right)^{-2}$, while small wavenumbers are 
approximately unchanged. For comparison, note that a low-pass filter defined by averaging over a circle is equivalent to scaling the Fourier coefficients by a Bessel function of the first kind $J_{0}\left(L_{f} k\right)$, and that the amplitude of this Bessel function decays as $\left(L_{f} k\right)^{-1 / 2}$ (with oscillation). Similarly, a low-pass filter defined by convolution with a Gaussian is equivalent to scaling the Fourier coefficients by a Gaussian, which decays faster than exponentially with $k$. Our elliptic filter is thus more scale local than the circular average but less so than a Gaussian filter. Although these considerations formally apply only in a periodic domain, the qualitative aspect of scale locality is transferred to nonperiodic domains. Finally, we note that Nadiga and Straub (2010) investigate eddy energetics in a barotropic, wind-driven basin using a spectral-truncation filter; this filter epitomizes scale locality but is not spatially local, which is consistent with their investigation of the area-integrated energy budget.

The disadvantage of our filter is that it is inhomogeneous: it does not commute with spatial derivatives; this is a problem endemic to spatial filters in domains with boundaries. To make analytical progress in deriving an energy budget one must commute the filter with derivatives, which incurs errors. These errors are tracked in our diagnostic code and are generally limited to the region within a distance $L_{f}$ from the boundary. Because using different boundary conditions on the elliptic inversion results in changes primarily near the boundaries where errors are already large, the use of a different boundary condition like $\bar{q}_{i}=q_{i}$ would have a negligible effect on our results. Analysis of the viscous dissipation requires formally commuting the spatial filter with several derivatives; because commuting the filter with derivatives incurs errors, we choose to minimize these errors by using Laplacian instead of higher-order dissipation.

To alleviate the difficulties introduced by the inhomogeneity, we define the eddy streamfunction $\tilde{\psi}_{i}$ to be the solution of

$$
\begin{aligned}
& q_{1}^{\prime}=\nabla^{2} \tilde{\psi}_{1}+F_{1}\left(\tilde{\psi}_{2}-\tilde{\psi}_{1}\right)-F_{0} \tilde{\psi}_{1} \quad \text { and } \\
& q_{2}^{\prime}=\nabla^{2} \tilde{\psi}_{2}+F_{2}\left(\tilde{\psi}_{1}-\tilde{\psi}_{2}\right),
\end{aligned}
$$

with a boundary condition on the eddy streamfunction inversion $\tilde{\psi}_{i}=0$; this guarantees that the eddy velocity normal to the boundaries is zero. The more common mass-conserving integral boundary condition would introduce a large-scale barotropic component to $\tilde{\psi}_{i}$ (associated with the finite barotropic deformation radius), which is undesirable. In addition, the effect of massconserving boundary conditions on the baroclinic component of $\tilde{\psi}_{i}$ would be confined to the boundaries, where errors are already large. The eddy velocity $\tilde{\mathbf{u}}_{i}$ and vorticity $\tilde{\omega}_{i}$ are defined by $\tilde{\mathbf{u}}_{i}=\left(-\partial_{y} \tilde{\psi}_{i}, \partial_{x} \tilde{\psi}_{i}\right)$ and $\tilde{\omega}_{i}=\nabla^{2} \tilde{\psi}_{i}$. Note that $\psi_{i}^{\prime} \neq \tilde{\psi}_{i}, \mathbf{u}_{i}^{\prime} \neq \tilde{\mathbf{u}}_{i}$, and $\tilde{\omega}_{i} \neq \omega_{i}^{\prime}$, but the errors are concentrated near the boundaries.

The instantaneous eddy energy budget for an inhomogeneous spatial filter is

$$
\frac{1}{2} \partial_{t} \tilde{E}=G+F+D+\chi,
$$

where the eddy energy density is

$$
\tilde{E}=\frac{\rho_{0} f_{0}^{2}}{g^{\prime}}\left(\tilde{\psi}_{1}-\tilde{\psi}_{2}\right)^{2}+\frac{\rho_{0} f_{0}^{2}}{g} \tilde{\psi}_{1}^{2}+\rho_{0} \sum_{i}\left(H_{i}\left|\tilde{\mathbf{u}}_{i}\right|^{2}\right),
$$

the local generation rate due to wind forcing and interactions with the mean is

$$
G=-\rho_{0} H_{1} \tilde{\psi}_{1} F_{w}^{\prime}+\rho_{0} \sum_{i} H_{i} \tilde{\mathbf{u}}_{i} \times\left(\mathbf{u}_{i} q_{i}\right)^{\prime}
$$

the local dissipation rate by Ekman friction and viscosity is

$$
D=-\rho_{0} r H_{2}\left|\tilde{\mathbf{u}}_{2}\right|^{2}-\rho_{0} A_{h} \sum_{i} H_{i} \tilde{\omega}_{i}^{2},
$$

the nonlocal divergence of the energy flux is $F$, and $\chi$ is the collection of terms that result from inexact commuting of the filter with spatial derivatives. Details of the derivation and the precise forms of $F$ and $\chi$ are included in the appendix.

The general form of the energy budget derived using a temporal filter (specifically, a long-time average) instead of a spatial filter is similar, except that it does not include local eddy generation by the steady wind forcing and it does not include filter error because the temporal filter commutes with spatial derivatives. Details of the mean and eddy energy budgets defined for a time filter can be found in Holland (1978). Although the form of the temporal-filter budget is similar to that of the spatialfilter budget, the budgets themselves can be different for the reasons described above (e.g., large-scale Rossby waves appear in the mean of the spatial filter but in the eddies of the temporal filter). Furthermore, the spatialfilter budget is instantaneously meaningful, in contrast to the temporal-filter budget.

\section{Experimental setup}

\section{a. Basin simulations}

The numerical experiments are performed using a model based on the QG equations truncated to two layers, Eqs. (1) and (2). The free-slip conditions appropriate for 
harmonic viscosity are imposed at lateral walls: $\partial_{n}^{2} \psi=0$, where $\partial_{n}$ is the derivative normal to the wall. A third-order Adams-Bashforth scheme is used for time derivatives, centered differencing in space, Arakawa (1966) for the Jacobians, and a multigrid method for the elliptic inversions. Mass conservation is achieved by assuming that the domain-averaged layer thicknesses are constant (McWilliams 1977); this is imposed as a boundary condition on the potential vorticity inversion, Eqs. (3) and (4).

We perform experiments in a square domain of width $L_{x}=L_{y}=6144 \mathrm{~km}$ with a $20-\mathrm{km}$ baroclinic deformation radius. A large domain scale is chosen to allow a large interior area free of filter errors, because these are concentrated near boundaries, and to allow a large scale separation between the eddies and the gyres. The filter scale $L_{f}=180 \mathrm{~km}$ is chosen to be as small as possible given the constraint that only the gyres show up in the large-scale-mean streamfunction. Note that $L_{f}$ is defined as an inverse wavenumber, so, for example, jets with a peak-to-trough distance of $\pi L_{f} \approx 565 \mathrm{~km}$ would lie at the filter scale and jets with a peak-to-trough distance of $L_{f}=180 \mathrm{~km}$ would lie well within the eddy range of scales.

We perform experiments using two different windforcing profiles: zonal and nonzonal. In the experiments using zonal wind, the forcing takes the form

$$
F_{w}=\left\{\begin{array}{ll}
-\frac{\tau_{0}}{a} \sin \left[\frac{2}{a}\left(y-\frac{L_{y}}{9}\right)\right] & \text { for } \quad y \in\left[L_{y} / 9, L_{y}\right] \\
0 & \text { for } y \in\left[0, L_{y} / 9\right)
\end{array},\right.
$$

where $a=8 L_{y} / 9 \pi$. Note that there is no wind forcing in the southernmost $1 / 9$ of the domain. This makes for slightly more interesting asymmetric dynamics and allows some nonzonality in the westward flow of the subtropical gyre.

The nonzonal-wind forcing takes the form

$$
F_{w}=-\frac{\tau_{0}}{b} \sin \left[\frac{2 y}{b}-f(x, y)\right],
$$

where $b=L_{y} / \pi$ and $f(x, y)=\left(2 L_{y} / b\right)\left(0.8 x / L_{x}-0.4\right) \sin$ $(y / b)$. The amplitude of the zonal and nonzonal wind stress are both $\tau_{0}=0.4 \mathrm{~N} \mathrm{~m}^{-2}$, which is much larger than the average zonal wind over the midlatitudes. However, considering that the wind stress curl is scaled by a large meridional extent $L_{y}$ in Eqs. (12) and (13), this gives a moderate forcing large enough to produce eddies in the eastern half of the domain. The nonzonal form of the wind forcing follows Rhines and Schopp (1991) and is
TABLE 1. Model parameters.

\begin{tabular}{lcc}
\hline \multicolumn{1}{c}{ Parameter } & Symbol & Value \\
\hline Rossby deformation radius & $L_{\rho}$ & $20 \mathrm{~km}$ \\
Horizontal resolution & $\Delta x=\Delta y$ & $8 \mathrm{~km}$ \\
Domain scale & $L_{x}=L_{y}$ & $6144 \mathrm{~km}$ \\
Top layer & $H_{1}$ & $1000 \mathrm{~m}$ \\
Bottom layer & $H_{2}$ & $4000 \mathrm{~m}$ \\
Wind stress amplitude & $\tau_{0}$ & $0.4 \mathrm{~N} \mathrm{~m}^{-2}$ \\
Coriolis parameter & $f_{0}$ & $9.3 \times 10^{-5} \mathrm{~s}^{-1}$ \\
Beta parameter & $\beta$ & $1.74 \times 10^{-11} \mathrm{~m}^{-1} \mathrm{~s}^{-1}$ \\
Gravitational acceleration & $g$ & $9.81 \mathrm{~m} \mathrm{~s}^{-2}$ \\
Reduced gravity & $g^{\prime}$ & $0.0043 \mathrm{~m} \mathrm{~s}^{-2}$ \\
Reference density & $\rho_{0}$ & $1028 \mathrm{~kg} \mathrm{~m}^{-3}$ \\
Bottom friction coefficient & $r$ & $10^{-7} \mathrm{~s}^{-1}$ \\
Dissipation coefficient & $A_{h}$ & $50 \mathrm{~m}^{2} \mathrm{~s}^{-1}$ \\
Spatial-filter scale & $L_{f}$ & $180 \mathrm{~km}^{2}$ \\
\hline
\end{tabular}

designed to produce a nonzonal intergyre jet. A summary of the model parameters can be found in Table 1 .

Once the equilibrium is reached, the terms in the eddy energy budget are evaluated five times per day and are averaged over a period of 10 years. Although the budget is well defined instantaneously, we find that the smoothing effect of a 10 -yr average clarifies the results.

\section{b. Doubly periodic simulations}

As an independent measure of locality, we compare the local eddy kinetic energy density from the basin simulations to the kinetic energy density from a set of doubly periodic simulations, each using the same model parameters, and driven by the local baroclinic shear at each coarse-grained point in the domain. Specifically, we compute the large-scale streamfunction from the two primary gyre simulations by averaging $\Psi_{i} \equiv \psi_{i}-\tilde{\psi}_{i}$ over a period of 10 years. This is used to compute the baroclinic shear $U(\cos \theta, \sin \theta)=\nabla^{\perp}\left(\Psi_{1}-\Psi_{2}\right)$, where $U$ is the magnitude of the shear, $\nabla^{\perp}=\left(-\partial_{y}, \partial_{x}\right)$, and $\theta$ is its counterclockwise angle from eastward. A joint histogram of $U$ and $\theta$ (from both simulations) is used to determine the range of shear magnitudes and angles in the simulations, from which 48 representative combinations are chosen. Each of these is used to drive a doubly periodic simulation. The simulations, using a standard spectral model (described, e.g., in Smith 2007) at a resolution of $512^{2}$, use the same values of $F_{1}, F_{2}, \beta$, and $r$ as the primary gyre simulation (although the enstrophy dissipation is accomplished by a spectral filter, rather than biharmonic viscosity used in the gyre simulations). Each simulation is set in a domain of 10 Rossby wavelengths, and where possible, is brought to steady state; in cases with larger and very nonzonal shears, the simulations do not equilibrate. The results are discussed in section 4 , below. 

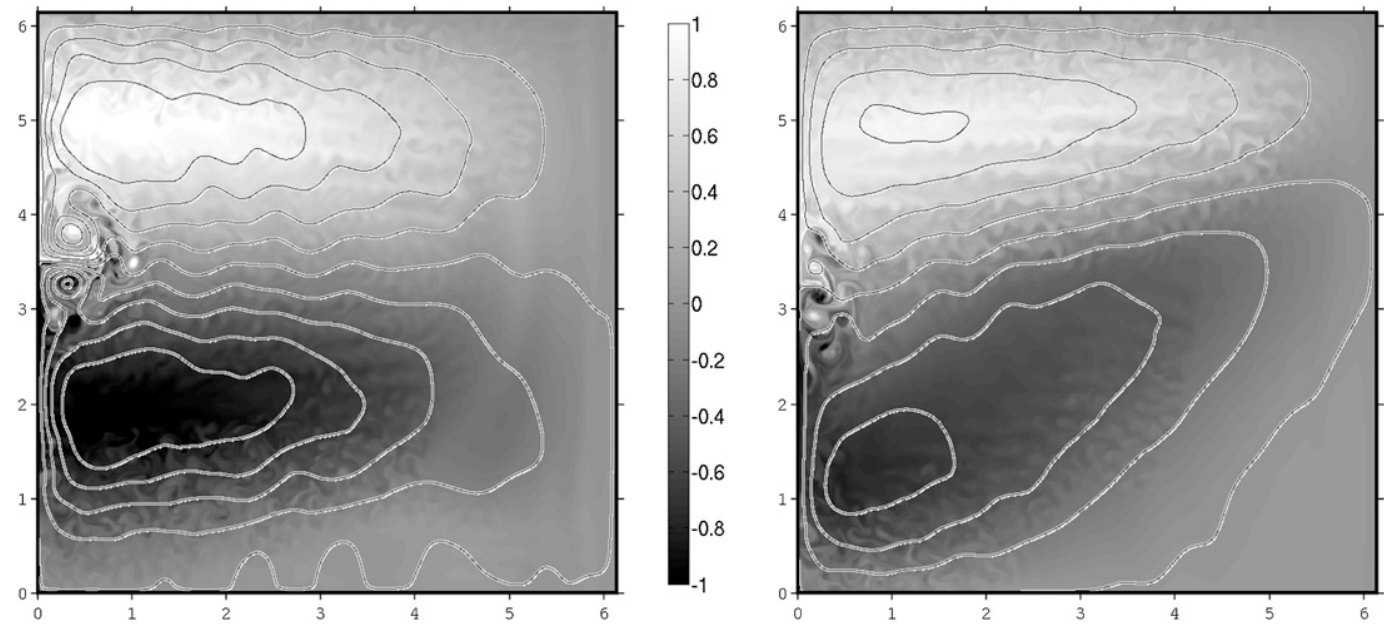

FIG. 1. Upper-layer potential vorticity and large-scale streamfunction. Snapshots of the upper-layer potential vorticity $q_{1}$ from the (left) zonal-wind and (right) nonzonal-wind experiments are overlaid by snapshots of the contours of large-scale upper-layer streamfunction $\psi_{1}-\tilde{\psi}_{1}$. The potential vorticity has been scaled by $10^{-4} \mathrm{~s}^{-1}$ and the streamfunction contour interval is $10^{4} \mathrm{~m}^{2} \mathrm{~s}^{-1}$. Axes in this and all subsequent maps are labeled in megameters.

\section{Results}

Figure 1 shows snapshots of upper-layer potential vorticity $q_{1}$ overlaid by contours of the instantaneous large-scale upper-layer streamfunction $\psi_{1}-\tilde{\psi}_{1}$. In the zonal-wind experiment (left), vigorous eddies are evident throughout the domain except the far east. Zonal jets are evident in the central part of the domain, but not the northern and southern quarters of the domain. In the nonzonal-wind experiment (right), eddies are evident in all regions except the southeast quarter. Strong zonal jets are evident in the eddy field superposed on the nonzonal large-scale intergyre jet. Because a spatial filter instead of a time average is used to separate mean and eddies, the instantaneous large-scale upper-layer streamfunction contours clearly show large-scale Rossby waves, but have almost no signature from the zonal jets. The use of a spatial filter also causes the western boundary currents to be slightly thicker than would be expected from a time-mean analysis.

Figure 2 shows the 10 -yr average of eddy kinetic energy density $\rho_{0} \sum_{i} H_{i}\left|\tilde{\mathbf{u}}_{i}\right|^{2}$ overlaid with contours of the baroclinic part of the 10 -yr-averaged large-scale streamfunction. In both experiments, the eddy kinetic energy
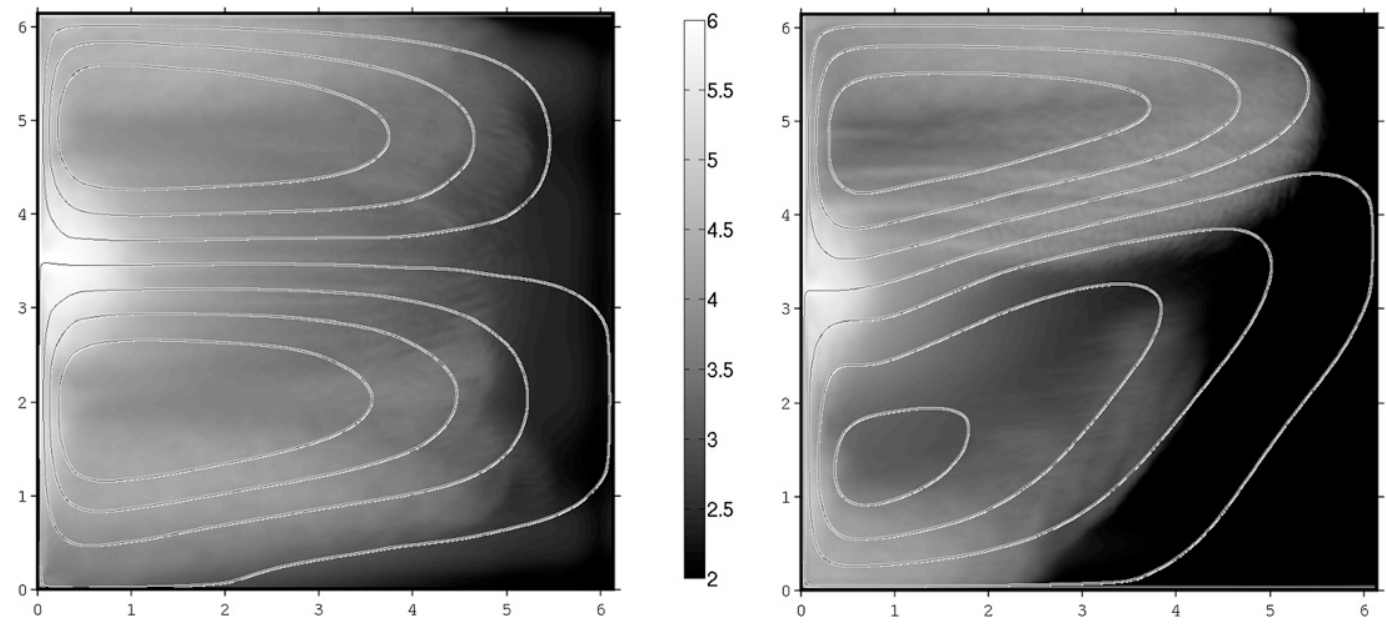

FIG. 2. Basin eddy kinetic energy density. The logarithm of the 10-yr average of eddy kinetic energy density $\left(\mathrm{kg} \mathrm{s}^{-2}\right)$, that is, $\log \left(\rho_{0} \sum_{i} H_{i}\left\langle\left|\tilde{\mathbf{u}}_{i}\right|^{2}\right\rangle_{t}\right)$, where $\langle\cdot\rangle_{t}$ denotes a 10 -yr average, is shown for the (left) zonal-wind and (right) nonzonal-wind experiments, overlaid by contours of the time-averaged large-scale baroclinic streamfunction, that is, $\left\langle\psi_{i}-\tilde{\psi}_{i}\right\rangle_{t}$. The contour interval is $10^{4} \mathrm{~m}^{2} \mathrm{~s}^{-1}$. 
varies over several orders of magnitude between the western boundary and the basin interior. Zonal jets are evident in the intergyre region of both experiments, though more weakly in the zonal-wind case. The zonalwind basin shows turbulent double-gyre flow, with almost no energy in the easternmost portion of the gyres and no jets. Nonzonal shear exists primarily in the eastern halves of the gyres, but also in the southwestward flow in the southern half of the subtropical gyre. The nonzonal-wind basin displays turbulent double-gyre flow but tilted on a northeast-southwest slant. The mean shear is nonzonal both in the intergyre flow and in the southeastern half of the subtropical gyre.

\section{a. Energy diagnostics and nonlocality}

Similar to the eddy kinetic energy, the terms in the energy budget vary by several orders of magnitude over the domain. To clarify the results, we present in Fig. 3 the terms in the energy budget scaled by the local eddy energy density for the basin simulations. The left column of Fig. 3 shows the zonal-wind results, and the right column shows the nonzonal-wind results. From top to bottom, the panels show the local generation, nonlocal terms, local dissipation, and filter error.

In both experiments there is a near cancellation between generation and flux, with weak overall dissipation; the energy budget is thus almost completely nonlocal from this perspective (we later reanalyze locality by coarse graining). Regions of blue generation indicate nonlinear transfer of energy from the eddies to the mean. Although such regions are found nearly everywhere, they are most pronounced in the region of the separating western boundary current, which presumably indicates a strengthening of the large-scale intergyre jet by the eddies. In order for a nonzero eddy energy level to be maintained in the presence of negative "generation," eddy energy must be fluxed in from elsewhere; this accounts for the strong influx of eddy energy to the western midlatitudes (indicated by red in the second row of Fig. 3). In both experiments the local eddy energy generation is more uniformly positive in the westward return flow of the gyres, and the local eddy dissipation is also stronger. This conspires to make the energetics more local, which is further verified by coarse graining, discussed below. The filter error, shown in the bottom row of Fig. 3, is negligible everywhere except in thin regions near the boundaries, though the error is somewhat more pronounced near the northern and southern edges of the western boundary.

Further quantification of the eddy energy budget is provided by Fig. 4, which plots the unscaled terms in the 10 -yr-averaged eddy energy budget for the zonal-wind case along the three lines of longitude shown in the lower left panel of Fig. 3; the lines lie at $x=L_{x} / 8, L_{x} / 4$, and $L_{x} / 2$.

Every panel of Fig. 4 shows significant variation with latitude. The leftmost panel shows the budget at $x=L_{x} / 8$, which cuts through the high-energy region just downstream of the boundary current separation. The terms in the budget in this region are an order of magnitude larger than anywhere to the east, and the budget is strongly nonlocal because of loss of eddy energy to the mean. This energy loss is compensated by a strong import of energy from the western boundary. The middle panel shows the budget $768 \mathrm{~km}$ further east at $x=L_{x} / 4$; the terms in the budget in this region are an order of magnitude smaller than in the leftmost panel. The westward flow in the north and south is approximately local because generation is positive and approximately balances dissipation. In the central eastward flow the generation is primarily negative and three jetlike structures are observed, with a strong nonlocal influx of energy. It is tempting to compare the budgets of these structures with theories of eddy-mean interactions in jet formation, but such theories inevitably treat the jets as part of the mean flow whereas both the jets and the waves of such theories are small enough to be part of our eddy field. The three bumps in the center panel of Fig. 4 have a wavelength of approximately $500 \mathrm{~km}$, which is much less than the cutoff wavelength of the filter $2 \pi L_{f} \approx 1130 \mathrm{~km}$. The rightmost panel shows that the terms in the eddy energy budget are quite small by $x=L_{x} / 2$; indeed, the terms in the central latitudes are probably "noise" rather than a true signal and would deteriorate further under a longer-time average. Thus, very little net generation, dissipation, or flux is taking place in the central part of the domain. Nevertheless, there remains a signature of local balance between generation and dissipation at the northern and southern edges, in the gyres' westward return flow.

Clearly, a great deal of spatial variation remains despite the ten-year average; indeed, Fig. 4 indicates that nonlocality is the rule rather than the exception when considering the budget at a single grid point of the domain. To clarify the level of nonlocality, and consistent with the concept of subgrid-scale parameterization, we coarse grain the eddy energy budget by averaging it over square boxes of width $384 \mathrm{~km}$, leaving $16 \times 16$ averaged subdomains; the qualitative results are not changed by using boxes of size $256 \mathrm{~km}$. The level of nonlocality is then assessed by plotting the ratio of the coarse-grained nonlocal terms to the coarse-grained dissipation, shown as a percentage in Fig. 5 for both basin experiments. Any region with nonlocal terms greater than or equal to $50 \%$ of the dissipation are considered to have nonlocal energetics so the grayscale is saturated at $50 \%$ in Fig. 5 . Regions with an eddy energy density less than $10^{3} \mathrm{~kg} \mathrm{~s}^{-2}$ 

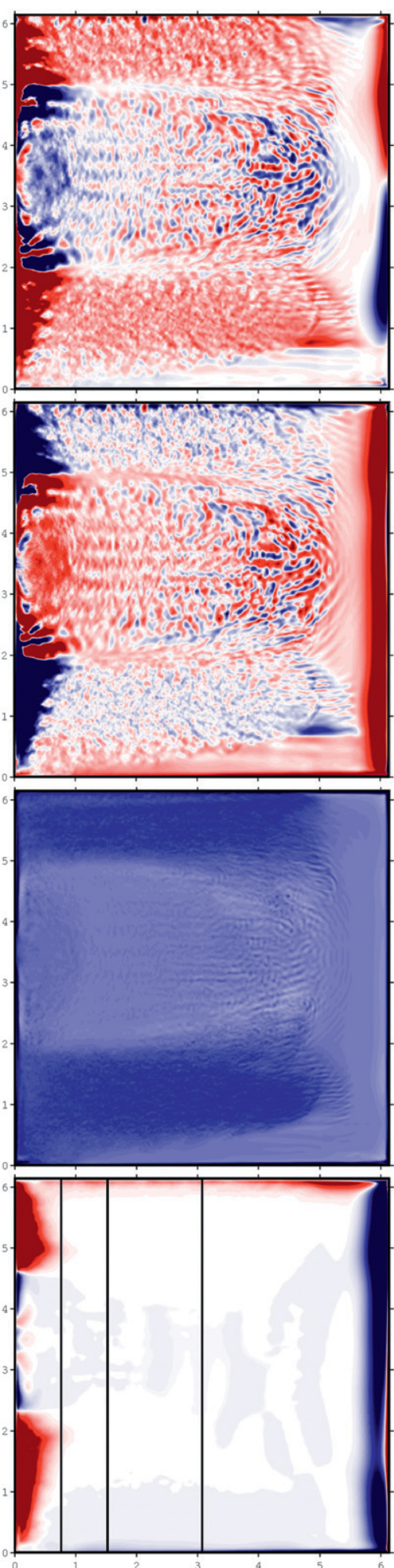
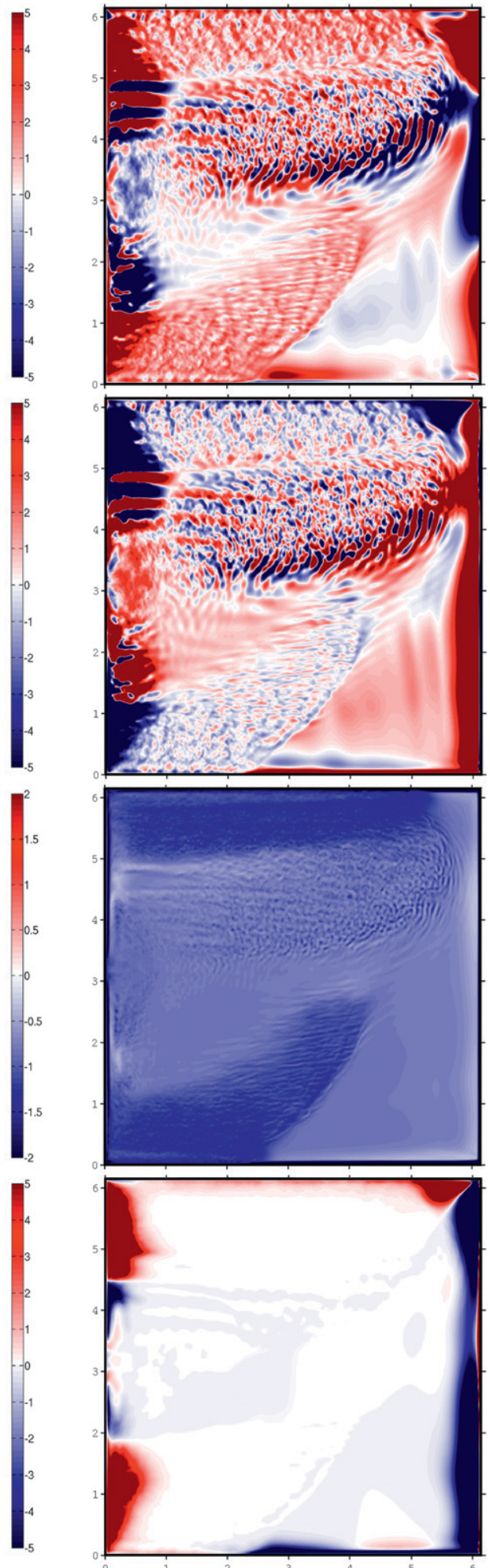

FIG. 3. Scaled terms in the eddy energy budget $\left(\mathrm{s}^{-1}\right)$. (from top to bottom) The scaled generation rate $\alpha\langle G\rangle_{t} /\langle\tilde{E}\rangle_{t}$, the scaled nonlocal flux rate $\alpha\langle F\rangle_{t} /\langle\tilde{E}\rangle_{t}$, the scaled dissipation rate $\alpha\langle D\rangle_{t} /\langle\tilde{E}\rangle_{t}$, and the scaled filter error rate $\alpha\langle\chi\rangle_{t} /\langle\tilde{E}\rangle_{t}$, where $\langle\cdot\rangle$ denotes a 10 -yr average and $\alpha=10^{7}$ is a scaling factor. The zonal-wind (left) and the nonzonal-wind (right) experiments are shown. 

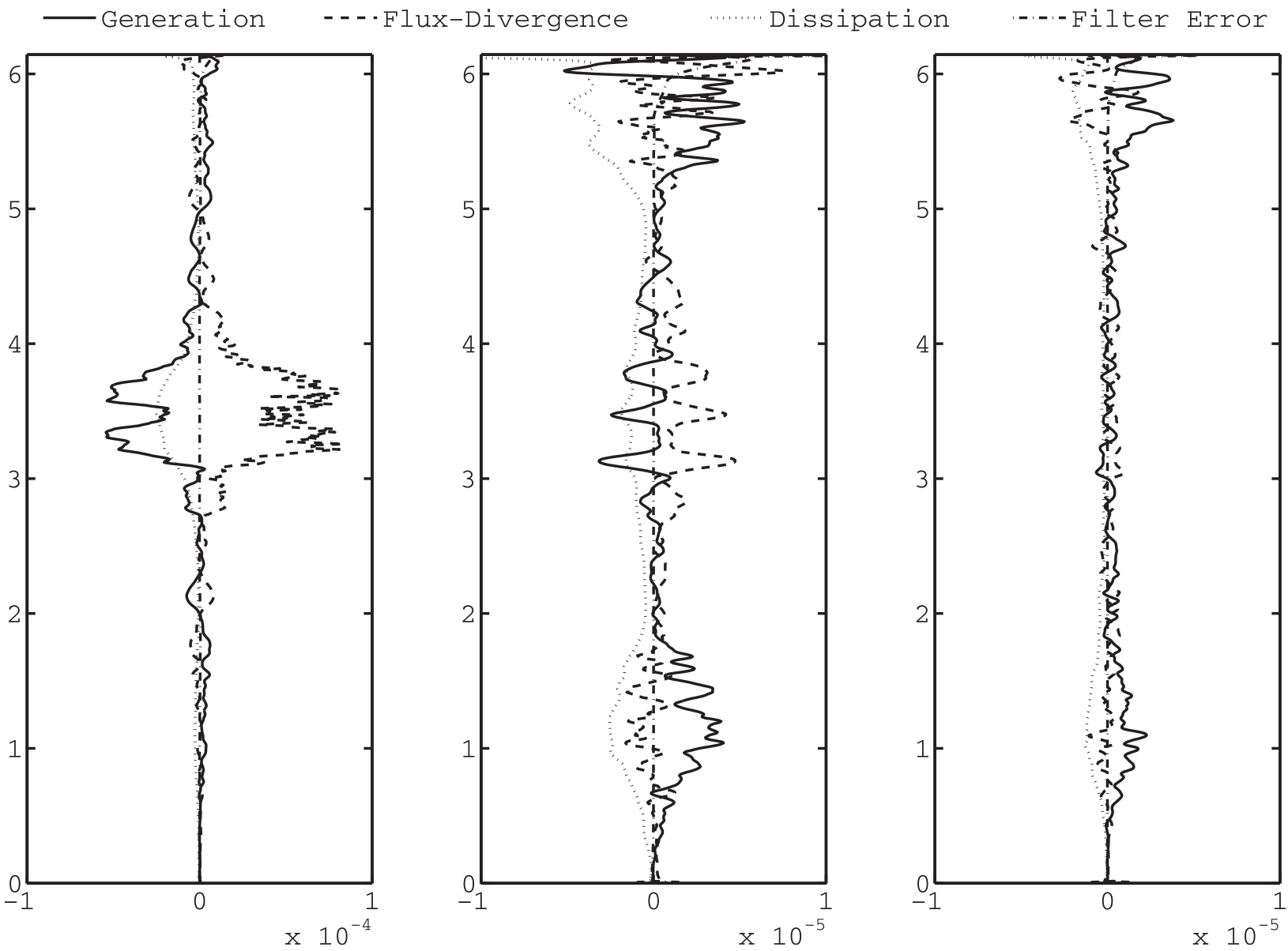

FIG. 4. Terms in the eddy energy budget $\left(\mathrm{m}^{3} \mathrm{~s}^{-3}\right)$. Plots of the terms in the 10-yr-average eddy energy budget of the zonal-wind case plotted along the lines of longitude shown in the bottom left panel of Fig. 3. (from left to right) The budget at $x=L_{x} / 8, L_{x} / 4$, and $L_{x} / 2$ is shown. The solid line is $\langle G\rangle_{t} / \rho_{0}$, the dashed line is $\langle F\rangle_{t} / \rho_{0}$, the dotted line is $\langle D\rangle_{t} / \rho_{0}$, and the dash-dot line is $\langle\chi\rangle_{t} / \rho_{0}$, where $\langle\cdot\rangle_{t}$ denotes a 10-yr average. The vertical axis measures the distance from the southern boundary in megameters.

are indicated by vertical lines, and regions with upscale energy transfer (i.e., where the coarse-grained local generation is negative) are indicated by horizontal lines. Note that horizontal lines can only appear in regions of strong nonlocality, shown in white in Fig. 5, although exceptions are possible immediately adjacent to the boundaries, because of contamination by filter error.

In both experiments the northern half of the subpolar gyre displays local energetics, indicated by dark shading (the boxes adjacent to the walls appear to be nonlocal, but are contaminated by filter error). In the zonal-wind experiment the southern half of the subtropical gyre is also approximately local despite some weak nonzonality in the large-scale baroclinic shear, but the central part of the basin displays strongly nonlocal energetics, with nonlocal terms depositing eddy energy in that region to make up for the losses due to dissipation and interaction with the large scales. In the nonzonal-wind experiment, the southeastern part of the subtropical gyre displays local energetics despite the nonzonal-mean shear.

\section{b. Comparison with doubly periodic simulations}

We assess the ability of doubly periodic simulations to model ocean patches with nonlocal energetics and/or with nonzonal-mean shear by performing doubly periodic simulations as described in section 3 . A range of 48 values of baroclinic shear are chosen to cover the observed range of large-scale, time-averaged shear in the basin simulations; the doubly periodic simulations are then mapped to patches of ocean in the basin simulations with similar shear profiles. Figure 6 shows the eddy kinetic energy density obtained from the 48 periodic simulations and mapped into the basin configuration; the left panel shows the zonal-wind experiment, and the right panel shows the nonzonal-wind experiment. 

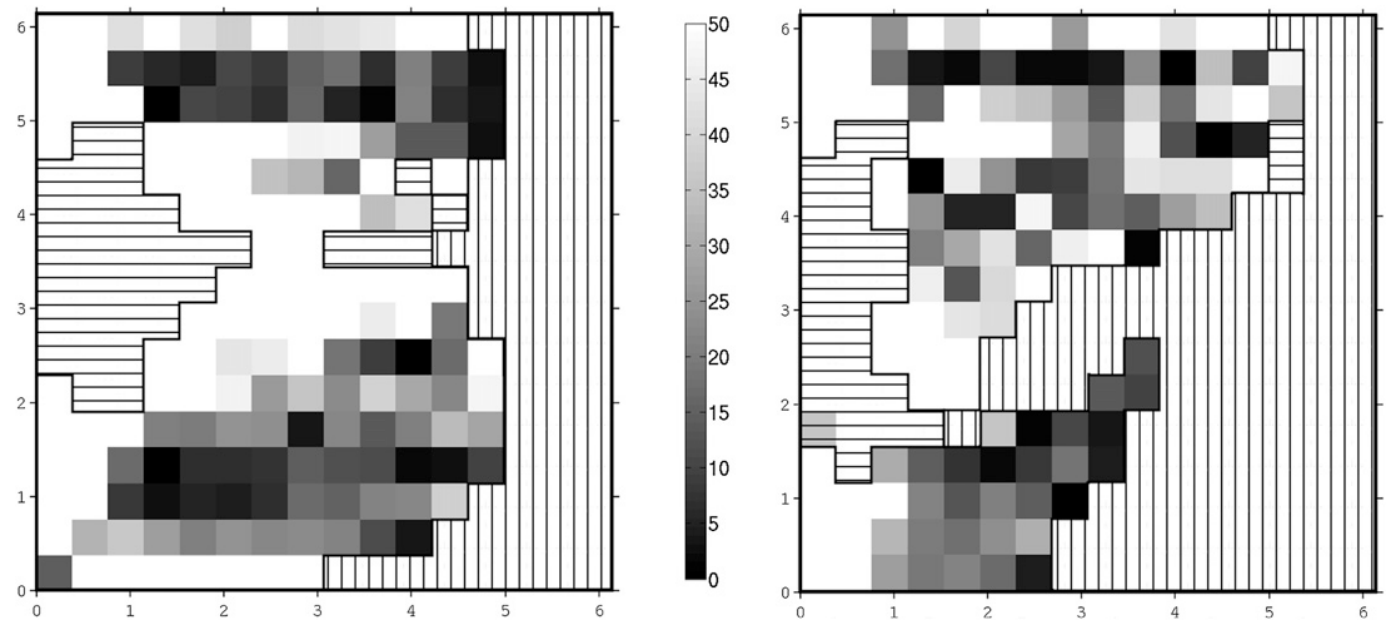

FIG. 5. Basin nonlocality. The ratio of coarse-grained nonlocal terms $\langle F\rangle_{t}$ to local dissipation $\langle D\rangle_{t}$ shown as a percentage for the (left) zonal-wind and (right) nonzonal-wind cases. Regions where the nonlinear energy transfer is upscale (negative generation $\langle G\rangle_{t}$ ) are indicated by horizontal lines. Regions where the coarse-grained energy density $\langle\tilde{E}\rangle_{t}$ is less than $10^{3} \mathrm{~kg} \mathrm{~s}^{-2}$ are indicated by vertical lines. Coarse-graining is performed by averaging over $16 \times 16$ square regions of width $384 \mathrm{~km}$, and $\langle\cdot\rangle_{t}$ denotes a 10 -yr average.

The results of the periodic simulations shown in Fig. 6 are clearly quite different from the basin results shown in Fig. 2. The most striking contrasts are evident in the central part of the basins and in the eastern edges of the gyres, where the eddy energy budgets are strongly nonlocal as shown in Fig. 5. In the central part of the basin the shear is predominantly zonal, but is subcritical and the doubly periodic simulations do not generate any eddy energy. In the eastern part of the gyres where the shear is strongly nonzonal the basin simulations have minimal eddy energy and strongly nonlocal budgets, whereas the doubly periodic simulations display the typical high-energy levels of simulations forced by strongly nonzonal shear. The regions of westward flow at the northern and southern edges of the basin display agreement between the eddy energy density of the doubly periodic and basin simulations, which is again consistent with the fact that the eddy energy budget is local in those regions (Fig. 5). The baroclinic shear in the southern half of the subtropical gyre has a nonzonal component in both zonalwind and nonzonal-wind experiments, yet the eddy energy budget in these regions is local, and the energy levels of the basin and periodic simulations are in agreement; thus, nonzonal shear does not always imply nonlocality.
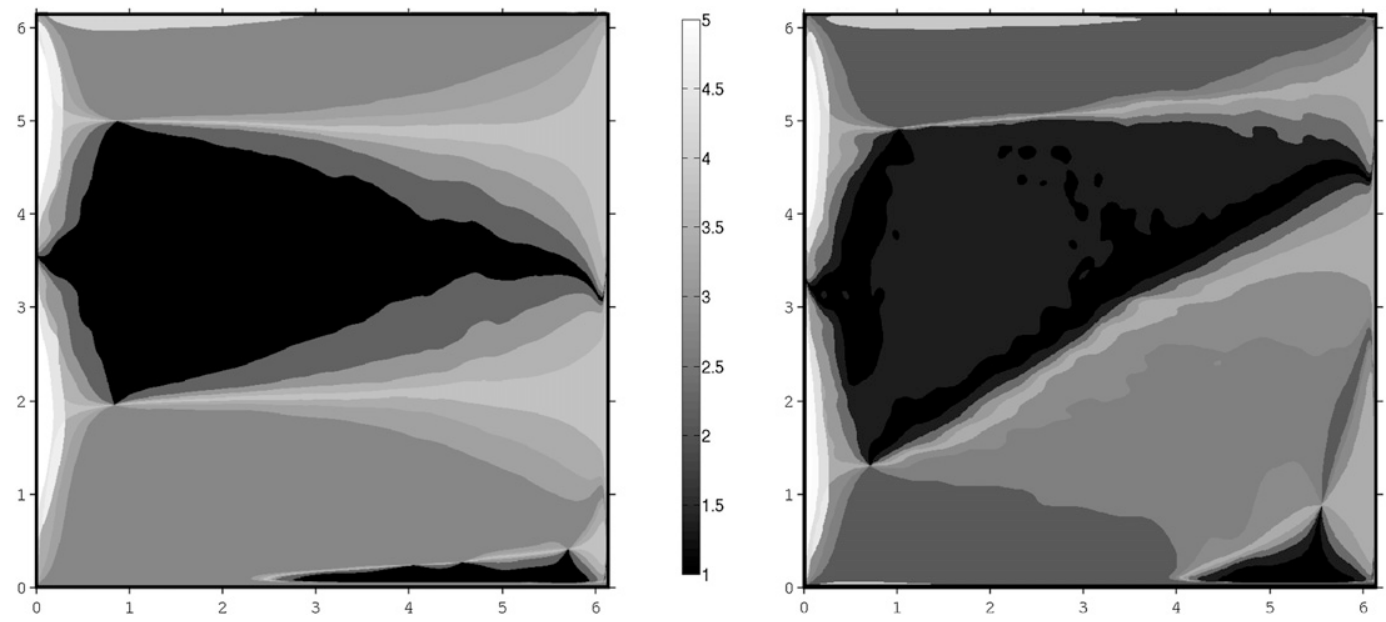

FIG. 6. Eddy kinetic energy density in periodic simulations. The logarithm of the average eddy kinetic energy density $\left(\mathrm{kg} \mathrm{s}^{-2}\right)$ from the suite of doubly periodic simulations is shown for the (left) zonal-wind and (right) nonzonalwind experiments. 
Finally, it is worth noting that the energy level of the doubly periodic simulations in the purely nonzonal western boundary current is comparable to that seen in the basin simulations. This result cannot be correlated with locality though, because the energy budget in the western boundary current is contaminated by filter errors and doubly periodic simulations are less relevant in direct proximity to the boundary.

\section{Discussion and conclusions}

This paper aims to diagnose the degree to which the energy budget of mesoscale eddies is local (i.e., to diagnose the degree to which it comprises a balance between local generation and dissipation) and to relate the degree of locality to the success of doubly periodic simulations. In contrast to most studies of energetics, we define the mean and eddies using a spatial filter instead of a time average, which connects more naturally to the concept of subgrid-scale parameterization. (Also note that our eddy field includes all small-scale features, including but not limited to nonlinear coherent vortices.) We derive the eddy energy budget and identify local (generation and dissipation) and nonlocal terms that consist of the divergence of an energy flux. The budget is then diagnosed in two simulations of wind-driven double-gyre simulations in square basins, one simulation with zonal-wind forcing and the other with nonzonal wind. Both simulations use two-layer quasigeostrophic dynamics.

A significant degree of nonlocality is found in both simulations, primarily in the intergyre jet region where eddies lose energy through nonlinear interactions with the mean. This energy loss and the loss caused by dissipation can only be balanced by a nonlocal import of eddy energy from elsewhere in the basin. The regions of westward flow to the north and south of the gyres display predominantly local energetics.

We run a suite of doubly periodic quasigeostrophic simulations using the same quasigeostrophic dynamics as the basin simulations, and forced by baroclinic shear taken from the basin simulations. The energy level in these doubly periodic simulations agrees with the eddy energy only in regions of the basin simulations that have local energetics, which is to say that the doubly periodic simulations are incompatible with ocean patches over approximately half the area of the basins. The periodic simulations generate far too little energy in the intergyre regions of the basin and far too much energy in the regions of nonzonal flow at the eastern edges of the gyres. It is not surprising that the doubly periodic simulations fail to model regions with nonlocal energetics; indeed, this can be viewed as confirming to some extent the validity of the results of the spatial-filter diagnostics.
It is well known that doubly periodic quasigeostrophic simulations forced by nonzonal baroclinic shear can develop unrealistically high energy levels (Arbic and Flierl 2004a; Smith 2007). Possible explanations are that such simulations have unrealistically low friction or require nonlinear friction (Arbic and Scott 2008), that they lack the effects of smaller-scale non-QG dynamics (Venaille et al. 2011), or that they lack the ability to export energy. By comparing patches of ocean with doubly periodic simulations using the exact same governing equations, we remove the possibility of incorrect friction or of non-QG dynamics. The failure of the doubly periodic simulations corresponding to regions of nonzonal shear at the eastern edges of the gyres and the diagnosed nonlocality of the eddy energy budget in these regions is therefore more likely to be caused by the inability of the doubly periodic simulations to export energy.

We note, though, that although the failure of some doubly periodic simulations with nonzonal shear can be linked to nonlocal energetics, it is not true that nonzonal shear is always associated with nonlocal energetics, or that zonal shear is always associated with local energetics. For example, the intergyre region in the zonalwind experiment has zonal baroclinic shear but nonlocal energetics. The southern edge of the subtropical gyre in both experiments, and in particular in the nonzonalwind experiment, has nonzonal baroclinic shear but local energetics. The only regions of nonzonal shear with nonlocal energetics are those at the eastern edges of the gyres where the baroclinic shear is weak and almost purely meridional; in these regions the inaccuracy of the doubly periodic simulations might also be explained by the presence of a strong barotropic shear that is absent from the periodic simulations. Alternatively, the inaccuracy of the doubly periodic simulations in these regions might be explained by failure to incorporate the time dependence of the large-scale background flow; Poulin et al. (2010) demonstrate that such time dependence can have a profound effect. We conclude that the eastern edges of the gyres cannot be correctly modeled by periodic simulations with time-independentmean shear, and a less idealized approach is called for (e.g., Spall 2000; Poulin et al. 2010).

The failure of doubly periodic simulations in Venaille et al. (2011) was difficult to attribute unambiguously because of the mismatch between the QG and primitive equation dynamics. The fact that in our experiments the success of doubly periodic simulations correlates well with energetic locality suggests that the failure of doubly periodic simulations in Venaille et al. (2011) is indeed an indication of nonlocal behavior rather than other possible causes (like time dependence of the mean flow, 
or a mismatch between $\mathrm{QG}$ and primitive equation dynamics).

Although our experiments are idealized and do not examine a range of forcing and drag parameters, our results suggest that eddy energy nonlocality is a potentially important feature of the ocean. Parameterizations, which allow nonlocality, like those of Eden and Greatbatch (2008) and Marshall and Adcroft (2010), may therefore be more successful in modeling the behavior of unresolved mesoscale eddies than parameterizations that assume locality. Similarly, because the degree of nonlocality in the real ocean is unknown, the validity of studies using doubly periodic simulations to interpret observations is uncertain (e.g., Arbic and Flierl 2004b; Arbic and Scott 2008).

Acknowledgments. I. G. is supported by the National Science Foundation (NSF) Collaboration in Mathematical Geosciences program, Grant DMS-1025468. L.-P. N. is supported by the Mathematics and Climate Research Network (MCRN) and by the Fonds québécois de la recherche sur la Nature et les Technologies (FQRNT). K. S. S. is partially supported by NSF Collaboration in Mathematical Geosciences program, Grant DMS1025468. Computer time was provided by the New York University (NYU) High Performance Computing facility and by the Courant Institute.

\section{APPENDIX}

\section{Derivation of the Eddy Energy Budget}

The derivation begins by applying the high-pass filter to the eddy PV Eqs. (1) and (2):

$\partial_{t} q_{1}^{\prime}=-\nabla \cdot\left(\mathbf{u}_{1} q_{1}\right)^{\prime}-\beta \tilde{v}_{1}+F_{w}^{\prime}+A_{h} \nabla^{4} \tilde{\psi}_{1}+$ Error $_{1}$ and

$\partial_{t} q_{2}^{\prime}=-\nabla \cdot\left(\mathbf{u}_{2} q_{2}\right)^{\prime}-\beta \tilde{v}_{2}-r \tilde{\omega}_{2}+A_{h} \nabla^{4} \tilde{\psi}_{2}+$ Error $_{2}$.

The Error $_{i}$ terms result from noncommutation of the filter with spatial derivatives; the relative RMS error in each layer, defined by

$$
\frac{\int \mid \text { Error }\left._{i}\right|^{2} d t}{\int\left|\partial_{t} q_{i}^{\prime}\right|^{2} d t},
$$

is tracked in the code and found to be less than 1\% except in a thin region near the boundaries.
The derivation proceeds by multiplying Eq. (A1) by $-\rho_{0} H_{1} \tilde{\psi}_{1}$, multiplying Eq. (A2) by $-\rho_{0} H_{2} \tilde{\psi}_{2}$, and adding the results. Subsequent simplifications are primarily applications of the chain rule in the form $\tilde{\psi} \nabla \cdot \mathbf{F}=$ $-\nabla \tilde{\psi} \cdot \mathbf{F}+\nabla \cdot(\tilde{\psi} \mathbf{F})$.

The local generation $G$ is defined by

$G=\rho_{0} \sum_{i}\left\{H_{i}\left[\tilde{u}_{i}\left(v_{i} q_{i}\right)^{\prime}-\tilde{v}_{i}\left(u_{i} q_{i}\right)^{\prime}\right]\right\}-\rho_{0} H_{1} \tilde{\psi}_{1} F_{w}^{\prime}$.

The terms corresponding to interaction with the mean and wind generation are tracked separately in the code, and the latter is found to be negligible.

The local dissipation $D$ is defined by

$$
D=-\rho_{0} r H_{2}\left|\tilde{\mathbf{u}}_{2}\right|^{2}-\rho_{0} A_{h} \sum_{i} H_{i} \tilde{\omega}_{i}^{2}
$$

and the Ekman and viscous dissipation terms are tracked separately in the code.

The nonlocal terms are equal to the divergence of an energy flux, defined as follows

$$
\begin{aligned}
F= & \rho_{0} \boldsymbol{\nabla} \cdot\left\{\frac{r H_{2}}{2} \nabla\left(\tilde{\psi}_{2}\right)^{2}+A_{h} \mathbf{F}_{A_{h}}\right. \\
& \left.+\sum_{i} H_{i}\left[\tilde{\psi}_{i} \partial_{t} \nabla \tilde{\psi}_{i}+\tilde{\psi}_{i}\left(\mathbf{u}_{i} q_{i}\right)^{\prime}+\beta \hat{\mathbf{x}} \tilde{\psi}_{i}^{2} / 2\right]\right\} .
\end{aligned}
$$

The terms corresponding to $\beta$, nonlinearity, and $\tilde{\psi}_{i} \partial_{t} \nabla \tilde{\psi}_{i}$ are tracked separately in the code, while the Ekman and viscous nonlocal terms are tracked as one term, because they are both small in comparison to the other terms. The divergence of the viscous-induced energy flux is defined by

$$
\nabla \cdot \mathbf{F}_{A_{h}}=\sum_{i} H_{i}\left(\tilde{\omega}_{i}^{2}-\tilde{\psi}_{i} \nabla^{4} \tilde{\psi}_{i}\right)
$$

The effect of filter error on the energetics is defined by

$$
\chi=-\rho_{0} \sum_{i} H_{i} \tilde{\psi}_{i} \text { Error }_{i} .
$$

\section{REFERENCES}

Arakawa, A., 1966: Computational design for long-term numerical integration of the equations of fluid motion: Two-dimensional incompressible flow. Part I. J. Comput. Phys., 1, 119-143.

Arbic, B. K., and G. Flierl, 2004a: Effects of mean flow direction on energy, isotropy, and coherence of baroclinically unstable beta-plane geostrophic turbulence. J. Phys. Oceanogr., 34, 77-93.

$\longrightarrow$, and $-2004 \mathrm{~b}$ : Baroclinically unstable geostrophic turbulence in the limits of strong and weak bottom Ekman friction: 
Application to midocean eddies. J. Phys. Oceanogr., 34, $2257-$ 2273.

—- and R. B. Scott, 2008: On quadratic bottom drag, geostrophic turbulence, and oceanic mesoscale eddies. J. Phys. Oceanogr., 38, 84-103.

Cessi, P., 2008: An energy-constrained parameterization of eddy buoyancy flux. J. Phys. Oceanogr., 38, 1807-1819.

Chelton, D. B., M. G. Schlax, R. M. Samelson, and R. A. de Szoeke, 2007: Global observations of large oceanic eddies. Geophys. Res. Lett., 34, L15606, doi:10.1029/2007GL030812

,$- \ldots$, and - 2011: Global observations of nonlinear mesoscale eddies. Prog. Oceanogr., 91, 167-216.

Eden, C., and R. J. Greatbatch, 2008: Towards a mesoscale eddy closure. Ocean Modell., 20, 223-239.

Ferrari, R., S. M. Griffies, A. J. G. Nurser, and G. K. Vallis, 2010: A boundary-value problem for the parameterized mesoscale eddy transport. Ocean Modell., 32, 143-156.

Gent, P. R., and J. C. McWilliams, 1990: Isopycnal mixing in ocean circulation models. J. Phys. Oceanogr., 20, 150-155.

Gill, A. E., J. S. A. Green, and A. J. Simmons, 1974: Energy partition in the large-scale ocean circulation and the production of mid-ocean eddies. Deep-Sea Res., 21, 499-528.

Grooms, I., K. S. Smith, and A. J. Majda, 2012: Multiscale models for synoptic-mesoscale interactions in the oceans. Dyn Atmos. Oceans, 58, 95-107.

Hall, M. M., 1986: A diagnostic investigation of kinetic energy budgets in a numerical model. J. Geophys. Res., 91 (C2), 25552568.

Harrison, D. E., 1979: Eddies and the general circulation of numerical model gyres: An energetic perspective. Rev. Geophys. Space Phys., 17, 969-979.

_ , and A. R. Robinson, 1978: Energy analysis of open ocean regions of turbulent flows-Mean eddy energetics of a numerical ocean circulation experiment. Dyn. Atmos. Oceans, 2 , 185-211.
Holland, W. R., 1978: The role of mesoscale eddies in the general circulation of the ocean-Numerical experiments using a wind-driven quasi-geostrophic model. J. Phys. Oceanogr., 8, 363-392.

Marshall, D. P., and A. J. Adcroft, 2010: Parameterization of ocean eddies: Potential vorticity mixing, energetics, and Arnold's first stability theorem. Ocean Modell., 32, 188-204.

McWilliams, J. C., 1977: A note on a consistent quasigeostrophic model in a multiply connected domain. Dyn. Atmos. Oceans, 1, 427-441.

Nadiga, B. T., 2008: Orientation of eddy fluxes in geostrophic turbulence. Philos. Trans. Roy. Soc., A366, 2491-2510.

— , and D. N. Straub, 2010: Alternating zonal jets and energy fluxes in barotropic wind-driven gyres. Ocean Modell., 33, 257-269.

Poulin, F. J., G. R. Flierl, and J. Pedlosky, 2010: The baroclinic adjustment of time-dependent shear flows. J. Phys. Oceanogr., 40, 1851-1865.

Rhines, P. B., and R. Schopp, 1991: The wind-driven circulation: Quasi-geostrophic simulations and theory for nonsymmetric winds. J. Phys. Oceanogr., 21, 1438-1469.

Smith, K. S., 2007: Eddy amplitudes in baroclinic turbulence driven by nonzonal mean flow: Shear dispersion of potential vorticity. J. Phys. Oceanogr., 37, 1037-1050.

Spall, M. A., 2000: Generation of strong mesoscale eddies by weak ocean gyres. J. Mar. Res., 58, 97-116.

Treguier, A. M., 1992: Kinetic energy analysis of an eddy resolving, primitive equation model of the North Atlantic. J. Geophys. Res., 97, 687-701.

Venaille, A., G. K. Vallis, and K. S. Smith, 2011: Baroclinic turbulence in the ocean: Analysis with primitive equation and quasigeostrophic simulations. J. Phys. Oceanogr., 41, 1605-1622.

Visbeck, M., J. Marshall, T. Haine, and M. Spall, 1997: Specification of eddy transfer coefficients in coarse-resolution ocean circulation models. J. Phys. Oceanogr., 27, 381-402. 